BNL-NCS-65687

IAEA-NDS-205

Informal Report

\title{
NuDat System for Access to Nuclear Data
}

\author{
Charles. L. Dunford \\ and \\ Robert R. Kinsey \\ National Nuclear Data Center \\ Brookhaven National Laboratory \\ Upton, New York, USA
}

July 7,1998

RECEIVED

Alls 171998

USTI

DISTRIBUTION OF THIS DOCUMENT IS UNLIMTED 


\section{DISCLAIMER}

This report was prepared as an account of work sponsored by an agency of the United States Government. Neither the United States Government nor any agency thereof, nor any of their employees, makes any warranty, express or implied, or assumes any legal liability or responsibility for the accuracy, completeness, or usefulness of any information, apparatus, product, or process disclosed, or represents that its use would not infringe privately owned rights. Reference herein to any specific commercial product, process, or service by trade name, trademark, manufacturer, or otherwise does not necessarily constitute or imply its endorsement, recommendation, or favoring by the United States Government or any agency thereof. The views and opinions of authors expressed herein do not necessarily state or reflect those of the United States Government or any agency thereof. 


\section{DISCLAIMER}

Portions of this document may be illegible electronic image products. Images are produced from the best available original document. 


\section{Contents}

1 Introduction 1

2 NUDAT data 1

3 NUDAT Options 2

4 Retrieval Strategy 2

5 SELECT 3

5.1 Data Selection Expressions $\ldots \ldots \ldots \ldots \ldots$

5.2 Parameter Value Specification . . . . . . . . . . . . . . . 9

6 RETRIEVE

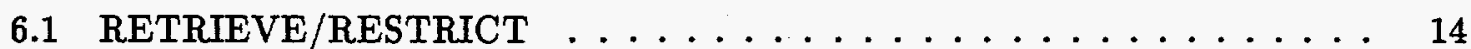

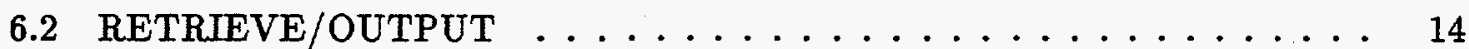

6.3 RETRIEVE/SEND . . . . . . . . . . . . . . . . 16

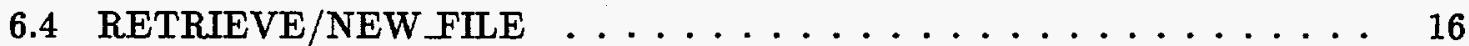

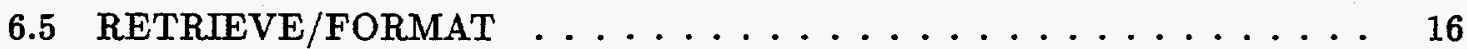

$\begin{array}{llr}7 & \text { FORMAT } & 16\end{array}$

8 NEW_FILE $\quad 16$

9 MASS_STATUS $\quad 17$

10 References $\quad 19$ 


\section{Introduction}

The NuDat program provides a user with access to nuclear properties and to some nuclear reaction data. The program operates on DEC VMS operating systems and on PC's with Microsoft operating systems. The program has four user interfaces, all having the same content and functionality. These interfaces are Web, Video and Sequential for VMS. The PC interface is identical to the VMS Video interface. Forms are used to supply the type of data the user desires, the retrieval parameters, the output format, and the sort order of the data.

The program and associated database is used in basic research, particularly for the systematic study of nuclear properties. It is also a useful tool for applied research to identify radiations from radionuclides contained in environmental samples, or from those produced by neutron or charged particle activation.

The NuDat database is derived from several databases maintained by the National Nuclear Data Center. The databases are the Adopted Levels and Gammas data sets from ENSDF[1],[2],[3],[4], the Nuclear Wallet Cards[5],[6], Decay Radiations derived from ENSDF decay data sets processed by RADLIST[7], and Thermal Neutron Cross Sections[8],[9].

\section{NUDAT data}

The Nuclear Data file, NUDAT, contains a variety of nuclear data in a form suitable for performing a wide range of searches for data satisfying user chosen constraints. The data base system is written using an ISAM-type data base so that the program will also operate on a PC. The data base contains the following information:

1. Level and gamma ray adopted properties of nuclides derived from the adopted data sets in the ENSDF data base [1].

2. Nuclear ground and metastable state properties as contained in the "Nuclear Wallet Cards"[5].

3. Radiations seen in radioactive decay of nuclides derived from the radioactive decay data sets in the ENSDF data base [7].

4. Thermal neutron cross sections and resonance integrals as published in Neutron Cross Sections, Vol $1[8,9]$.

The data contained in this data base are designed to be consistent with the contents of the ENSDF data base and are updated concurrently with that data base. In particular, the ground and metastable state properties are more current than those contained in the "Nuclear Wallet Cards" publication. The thermal neutron cross section and resonance integral data are the same as given in the publication since work in this area has been terminated. 


\section{NUDAT Options}

The main menu and the associated options are summarized in the following diagram. The options listed on the top line of the diagram represent the top-level menu. Where an option is itself a menu of sub-options, the secondary menu items are listed vertically below the top-level option. Each option and sub-option will be described in detail below. All top-level options will be given in the text all in capital letters; sub-options will also be given in capital letters with a slash between the option and sub-option. For example RETRIEVE and RETRIEVE/OUTPUT.

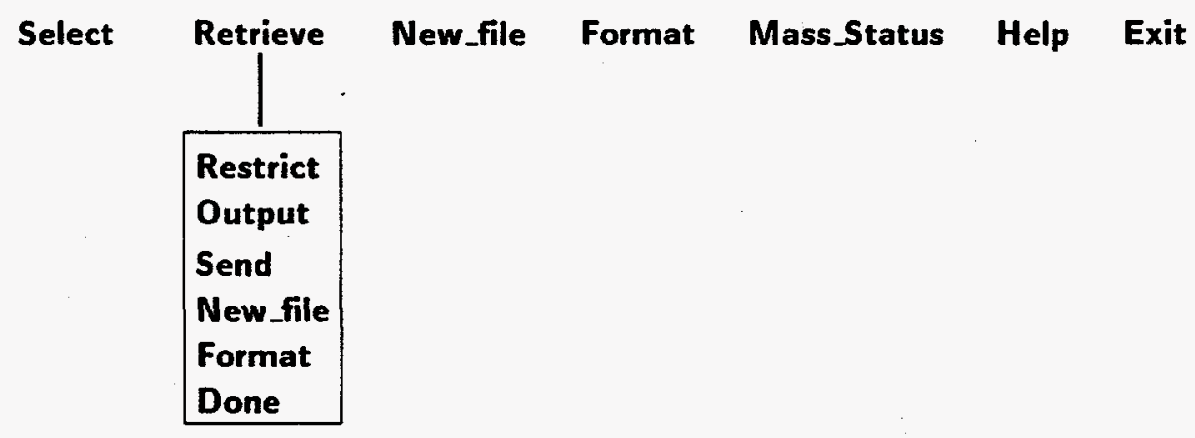

With the SELECT option, the user enters data selection values. Records satisfying the selection criteria are store for later use. This information or selected parts thereof may be retrieved with the RETRIEVE option. The stored information may be further narrowed with the RETRIEVE/RESTRICT. Output to terminal or disk file is accomplished with the RETRIEVE/OUTPUT option. The NEW FILE and FORMAT options select the output media and output format respectively. The MASS_STATUS option allows the user to view the publication status for selected mass-chains.

\section{Retrieval Strategy}

The SELECT option should be used to select the type of data desired. Depending on the type of data selected, the user must next supply values for the data retrieval parameters. Only the retrieval parameters appropriate for the selected data type are displayed. The user then enters the selection expression (see section 5.2) for the parameters on which selection is to be made. A blank entry means that no selection will be made on that parameter.

When all selection expressions have been supplied, the program will retrieve all data records which satisfy the selection criteria. The number of records containing the selected information is displayed and the sub-menu displayed. At this point one may either display the retrieved data on the user's terminal or store it in a disk file with the RETRIEVE option. The output can be ordered in several different ways depending on the type of data. The RETRIEVE/RESTRICT option can be used to select a subset of the retrieved data records. This subset of the data records can then be sorted and displayed. With the RETRIEVE/NEW_FILE option, you may specify whether terminal or disk output is desired; with the RETRIEVE/FORMAT option, you can select the output format. 


\section{SELECT}

The SELECT option which appears in the top-level menu should be used to choose the data and then to output the selected data to either the user's terminal or to a disk file. The data type is selected and the appropriate retrieval parameter expressions entered.

The data types which may be selected are the following:

- Levels - information about nuclear levels which includes the nucleus, the level energy and its uncertainty, spin-parity, and the half-life and its uncertainty.

- Gammas - information about nuclear gamma rays including their energy and uncertainty, source level energy, intensity, multipolarity, mixing ratio and conversion coefficient.

- Levels and Gammas - information about gamma rays with the addition of spinparity and half-life of the parent level.

- Wallet Cards - information about ground and meta-stable state properties of nuclei including level energy, mass excess and uncertainty, spin-parity, half-life and uncertainty, decay mode with branching ratio and $Q$-value. For naturally occurring nuclides, the abundance is given.

- Decay Radiations - information about the radioactive decay of nuclides including radiation type, energy, intensity and dose. For beta radiation both the median and endpoint energies are given.

- Neutron Data - information about thermal neutron cross sections, fission neutron multiplicities and resonance integrals.

The video mode data selection menu is illustrated in the next figure. 
Data Type Selection Display in Video Environment

Nuclear Data File - NUDAT

\section{SELECT}

Format : Wide Table

File : User Terminal

Base Collection :

Current Collection :

\section{SELECT DATA TYPE}

LEVELS - Adopted levels from ENSDF.

GAMMAS

- Adopted gamma rays from ENSDF.

LEVELS AND GAMMAS - Adopted levels and gamma rays from ENSDF.

WALLET CARDS

- Ground and Metastable State Properties.

DECAY RADIATIONS

NEUTRON DATA

DONE

- ENSDF decay data processed by MEDLIST.

- Thermal Data and Resonance Integrals from BNL325.

- Return to top-level menu without selecting.

Video

In video mode, the NUDAT module displays the previous figure which is a menu of data types. Use the UP and DOWN ARROW keys to move between the menu items to select the data type. The initial selection is the previously selected data type. Implement a selection by depressing the RETURN key. You can exit without selecting a data type by choosing the DONE option.

The data selection menu is followed by the parameter selection form illustrated in the following six figures. A different list of parameters for data selection appropriate to the selected data type is presented in the form. Each field is displayed along with its current value. The selection expression in any field can be changed by moving to that field with the UP-ARROW or DOWN-ARROW key and then entering the new value. At the bottom of the display is brief information on the form and contents required for data entry in the selected field. The last item in the form is an OK/QUIT toggle which can be changed by depressing the SPACE BAR. Entry of a RETURN key on this field will cause the data base to be searched for the selected data if the toggle is on OK or will return to the top-level menu if on QUTT. 


\section{SELECT}

\section{Retrieval Parameters for Levels}

Mass Number $\quad \mathbf{2 4 0 - 2 5 0}$

Element

Neutron Number

ODD/EVEN

E-level(keV)

Spin-Parity

$$
\begin{aligned}
& \text { E-E } \\
& -100.0 \& \sim 0.0
\end{aligned}
$$

Half-life

OK/QUIT

$$
\text { 2+ }
$$

OK

You may select the evenness or oddness of the nuclide's neutron and proton numbers. One or more values may be entered, separated by an \& for "AND" or an \& for "OR". A value can be negated with a $\sim$. Enter E-E for even-even, $\mathrm{O}-\mathrm{O}$ for odd-odd or E-O for even-odd.

Ex. E-E@O-O will select even-even or odd-odd nuclides.

Use UP- and DOWN-ARROWS to move between fields.

Levels

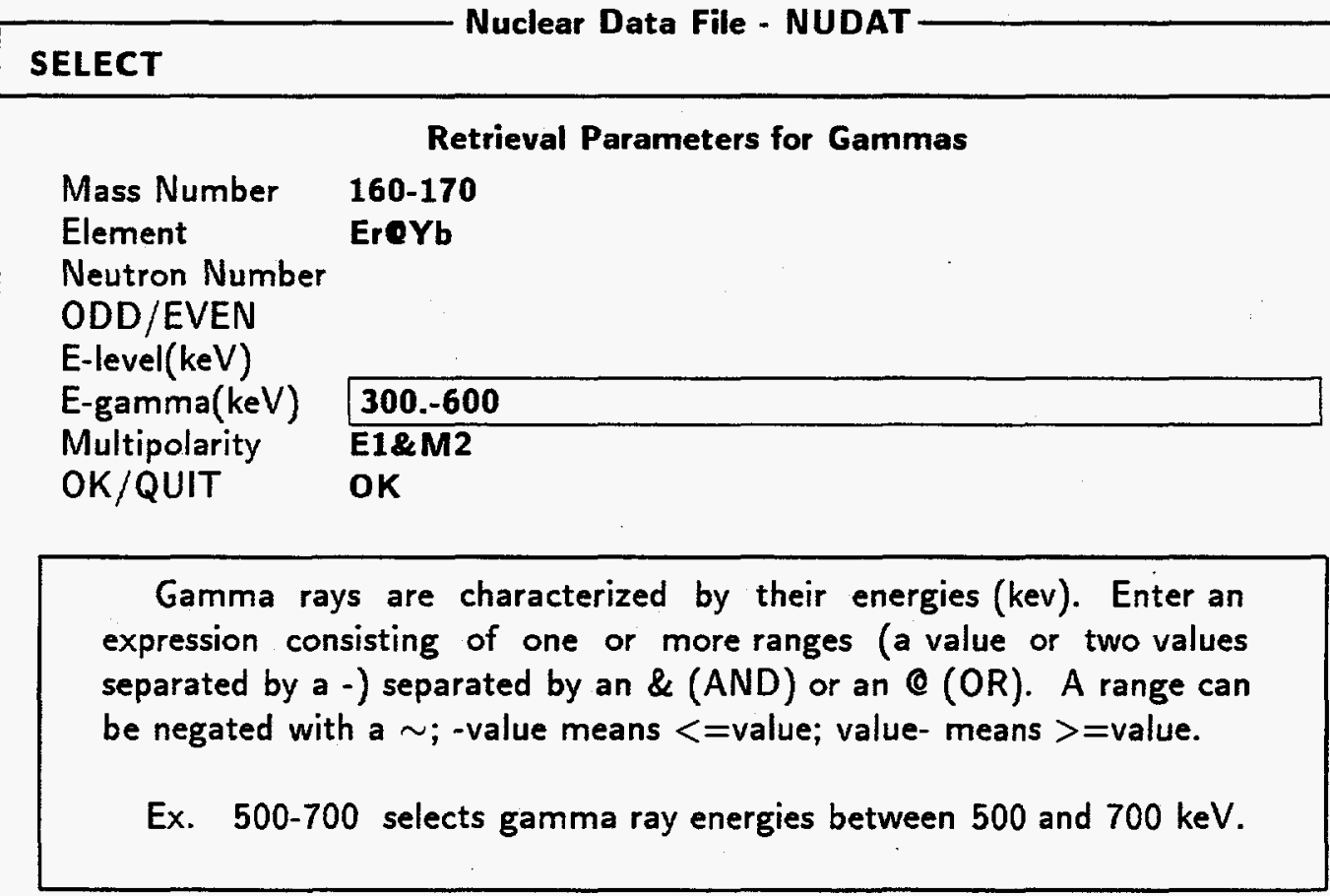

Use UP. and DOWN-ARROWS to move between fields.

\section{Gammas}


Parameter Selection in Video Environment (cont)

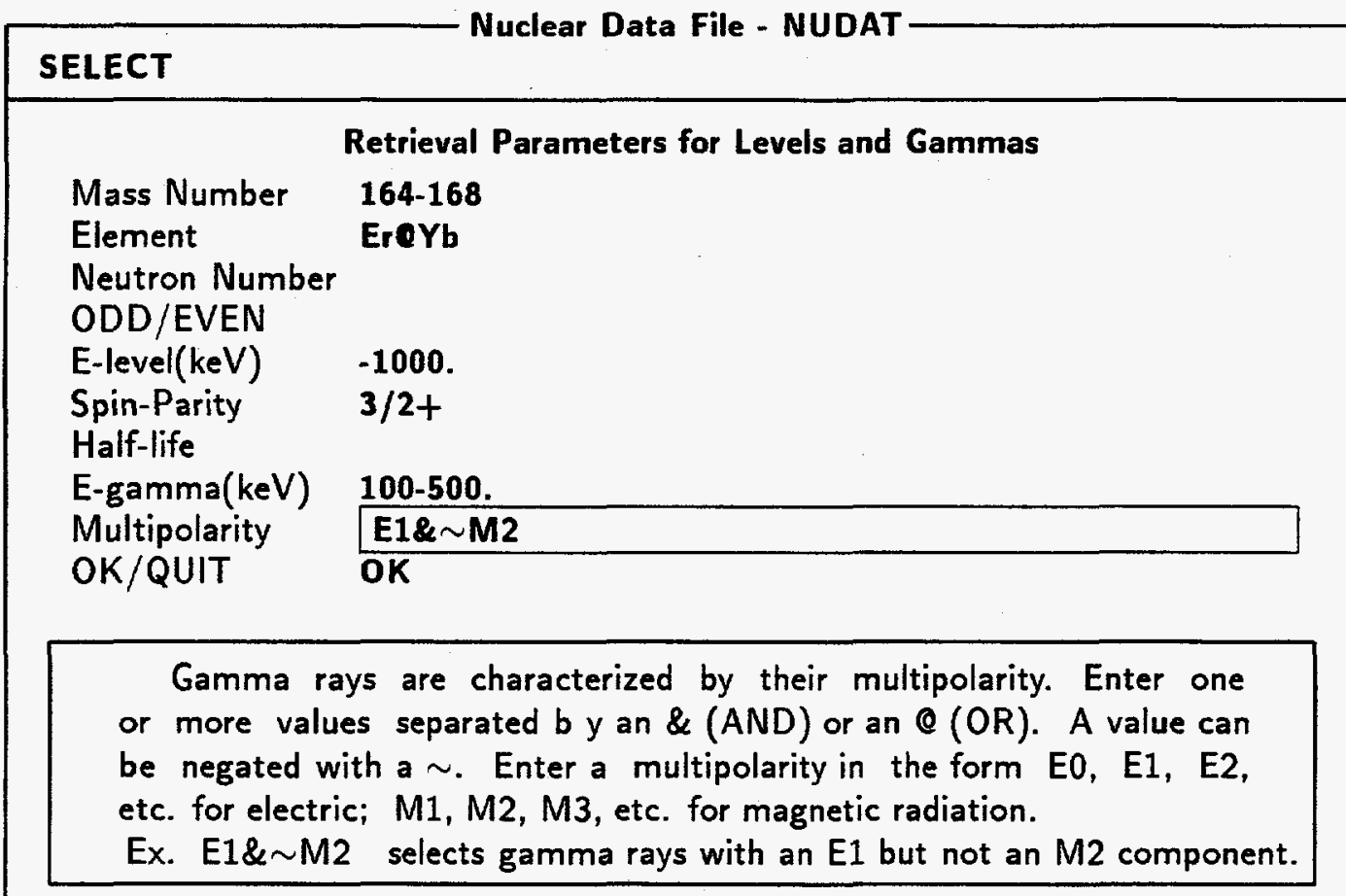

Use UP- and DOWN-ARROWS to move between fields.

Levels and Gammas

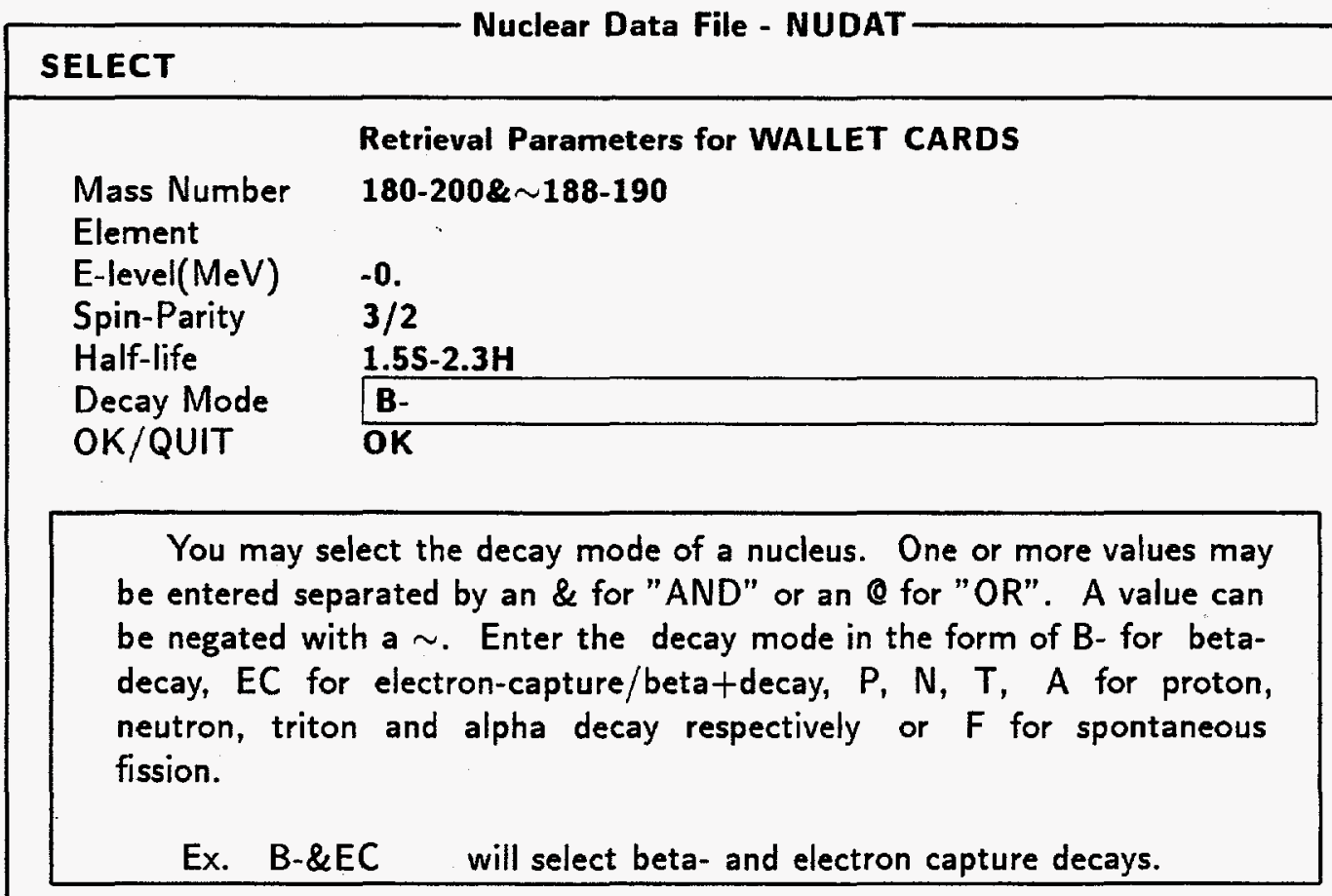

Use UP- and DOWN-ARROWS to move between fields.

WALLET CARDS 
Parameter Selection in Video Environment (cont)

Nuclear Data File - NUDAT

SELECT

Retrieval Parameters for Decay Radiations

Mass Number 1130115

Element In

Half-life

Decay Mode

Radiation

R-energy(keV)

R-intensity

OK/QUIT

\section{GQE}

35-

OK

You may select the radiation from a nuclide decay. One or more values may be entered separated by an \& for "AND" or an @ for "OR". A value can be negated with $a \sim$. Enter decay radiation in the form of $B$ - for beta-, B+ for positrons, $E$ for electrons, $G$ for gamma rays and $A$ for alpha particles.

Ex. $\mathrm{B}+\quad$ will select positron radiations.

Use UP- and DOWN-ARROWS to move between fields.

Decay Radiations

SELECT

Nuclear Data File - NUDAT

\begin{tabular}{|c|}
\hline $\begin{array}{l}\text { Mass Number } \\
\text { Element } \\
\text { Half-life } \\
\text { Reaction }\end{array}$ \\
\hline $\begin{array}{l}\text { Sigma } \\
\text { Resonance I. } \\
\text { OK/QUIT }\end{array}$ \\
\hline $\begin{array}{l}\text { Data may be selected by the value of thermal neutron cross } \\
\text { sections. Cross sections are given in barns. Enter a cross section } \\
\text { selection expression. This consists of one or more ranges separated } \\
\text { by an \& (AND) or an Q (OR). A range can be negated with a } \sim \text {. } \\
\text { A range is either a single value or two values separated by a }- \text { A } \\
\text { missing lower value (-A) means }<=A \text {; a missing upper value }(A-) \text { means } \\
>=\text { A. } \\
\text { Ex. 1.E+6- } \quad \text { will select cross sections }>=1 \text { million barns. }\end{array}$ \\
\hline
\end{tabular}

Use UP- and DOWN-ARROWS to move between fields.

Neutron Data 
In sequential mode, the NUDAT module will list the possible data types and a integer identification number. At the prompt, enter the identification number for the data type desired. A RETURN without a preceeding identification code will select the current default data type which is denoted by an * preceding the identification number. By entering a $D$ for DONE, the user is returned to the top-level menu.

The data selection menu is followed by the parameter selection form. A different list of parameters for data selection appropriate to the selected data type is presented in the form. Each field is displayed along with its current value and an integer identification number. At the prompt, select the fields to modify by entering the ID numbers separated by a comma. Entering ALL will select all parameters. You will be prompted for a new data selection expression for each chosen field one at a time. Enter a new expression, or a question mark (?) for brief information on the form and contents required for data entry in the selected field. A RETURN without any preceding characters will leave the field unchanged. After responding for all chosen fields, the parameter selection form appears again with updated parameter selection values. At this point, additional fields may be modified. Optionally, by entering DONE, the data base will be searched for the selected data, or by entering QUIT, the program will return to the top-level menu. See the preceding six figures which illustrate the video mode input form and which contain the retrieval parameters for each data type.

If no data satisfying the selection criteria are found, the user is informed and returned to the top-level menu. If data are found, the next menu is displayed as well as the number of data records found.

\subsection{Data Selection Expressions}

Data from the NUDAT data base are chosen by supplying a selection expression for one or more of the retrieval parameters. If no selection expression is supplied for a parameter, then it is assumed that all values for that parameter are desired. There are two types of selection expressions, one for numerical data and another for character.

An expression is made up of "ranges". These ranges are linked by logical operators, either an AND operator (\&) or an OR operator (@). Any range can be negated by preceeding it with a tilda character $(\sim)$. A general expression would have the form.

$$
\{\sim\} \text { range }_{1}\left\{\begin{array}{l}
@ \\
\&
\end{array}\right\}\{\sim\} \text { range }_{2}\left\{\begin{array}{l}
@ \\
\&
\end{array}\right\}\{\sim\} \text { range }_{3} \cdots
$$

An expression is evaluated from left to right. For example, to select data in range or in range $e_{2}$ but not in range then $_{3}$ enter

$$
\text { range }_{1} @ \text { range }_{2} \& \sim r a n g e_{3}
$$


A character variable range is just a single character string value for the parameter selection. A numeric variable range consists of a single numeric value or two numeric values separated by a minus sign (-). The four possible constructions are

$$
\begin{array}{ll}
\text { value }_{1} & \text { equal to value } \\
\text { value }_{1} \text {-value }_{2} & \text { between value } \\
\text { value }_{1} \text { - } & \text { greater than or equal to } \text { value }_{1} \\
\text { - }_{\text {value }} & \text { lees than or equal to value }
\end{array}
$$

For example to select values between 130 and 175 except for 150

$$
130-175 \& \sim 150
$$

\subsection{Parameter Value Specification}

There are 16 different parameters whose values can be specified in order to select data from this data base. Some of these data are essentially numeric in nature and some are character data. Not all parameters are appropriate for a selected data type. The detailed description of how to specify value for each different retrieval parameter is given in the following sections. These parameters are

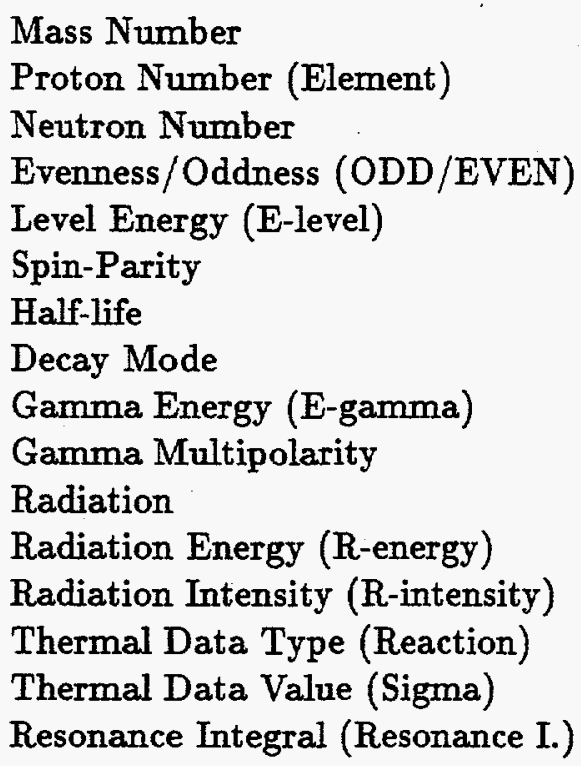

$\begin{array}{ll}\text { numeric } & \text { nuclide specification } \\ \text { numeric } & \text { nuclide specification } \\ \text { numeric } & \text { nuclide specification } \\ \text { character } & \text { nuclide property } \\ \text { numeric } & \text { level specification } \\ \text { character } & \text { level property } \\ \text { numeric } & \text { level property } \\ \text { character } & \text { level property } \\ \text { numeric } & \text { gamma ray specification } \\ \text { character } & \text { gamma ray property } \\ \text { character } & \text { radiation specification } \\ \text { numeric } & \text { radiation property } \\ \text { numeric } & \text { radiation property } \\ \text { character } & \text { thermal data specification } \\ \text { numeric } & \text { thermal data property } \\ \text { numeric } & \text { thermal data property }\end{array}$

Mass Number - A nuclide is characterized by the number of nucleons (protons and neutrons) in its nucleus. This is a numeric selection parameter. The data base contains information for nuclei with mass numbers between 1 and 266. For example

180-210\& 200 selects nuclides with mass numbers between 180 and 210 but not equal to 200 . 
Proton Number - A nuclide is characterized by the number of protons (which corresponds to a chemical element) in its nucleus. This is a numeric selection parameter. The data base contains information for nuclei with proton numbers between 0 and 109. Chemical symbols may also be entered instead of the equivalent proton number. For example

U@94- $\quad$ selects elements with proton numbers 92 (Uranium) and 94 and above.

Neutron Number - A nuclide is characterized by the number of neutrons in its nucleus. This is a numeric selection parameter. The data base contains information for nuclei with neutron numbers between 0 and 160 . For example

57-77@82-88 selects nuclides.with neutron numbers between 75 and 77 or between 82 and 88 .

Evenness/Oddness - A nuclide is characterized by the evenness or oddness of its neutron number and its proton number. This is a character selection parameter. The parameter may have only three values $\mathrm{E}-\mathrm{E}$ for even-even nuclides, $\mathrm{O}-\mathrm{O}$ for odd-odd nuclides, and $\mathrm{E}-\mathrm{O}$ for even-odd or odd-even nuclides. For example

E-E@O-O selects nuclides with even neutron numbers and even proton numbers or odd neutron numbers and odd proton numbers.

Level Energy - A level of a nucleus is determined by its excitation energy. This is a numeric selection parameter. Energies must be given in $\mathrm{keV}$. The ground state of a nucleus has an energy of 0.0 . For example

-2500 selects nuclear levels less than or equal to $2.5 \mathrm{MeV}$.

Spin-Parity - A level of a nucleus is characterized by its spin and parity. This is a character selection parameter. Spins are either integral (eg. 3) or half integral (eg. 3/2) and are given in that manner. The parity of a level may be either positive or negative. This parameter may be given as spin only, parity only or as spin and parity in the form $3+$ or 3/2-. If the input spin-parity is followed by an integer enclosed in square brackets ([n]), then only the $\mathrm{n}^{\text {th }}$ level with the requested spin-parity will be selected. For example

2@3- $\quad$ selects nuclear levels with spin 2 (any parity) or 3spin and parity.

$2+[1] \quad$ selects the first level in a nuclide $2+$ spin and parity. 
Half-life - A level of a nucleus can be characterized by its half-life. This is a numeric selection parameter. It is specified as a floating point number followed by one or more characters to indicate the time unit. The possible time units are

$\begin{array}{llll}S-\text { second } & \text { MS }- \text { millisecond } & \text { EV }- \text { electron volts } \\ M-\text { minute } & \text { US }- \text { microsecond } & \text { KEV }- \text { kilo electron volts } \\ H-\text { hour } & \text { NS }- \text { nanoseconds } & \text { MEV }- \text { million electron volts } \\ D-\text { day } & \text { PS }- \text { picoseconds } & \\ Y-\text { year } & \text { FS }- \text { femtoseconds } & \end{array}$

For stable nuclide states, one may enter STABLE. For example

3S-4H@STABLE selects nuclear states with half-lives between 3 seconds and 4 hours or ones that are stable against decay.

Decay Mode - The decay mode of a nuclear state may be selected. This is a character selection parameter. The possible decay modes and their character codes are

$$
\begin{aligned}
& \text { B- - beta- decay } \\
& \text { EC - electron-capture/positron decay } \\
& \text { P - proton decay } \\
& \text { N }- \text { neutron decay } \\
& \text { T }- \text { triton decay } \\
& \text { A - alpha decay } \\
& \text { F - spontaneous fission }
\end{aligned}
$$

Use these character codes to select the decay mode. For example

$$
\text { B- } \quad \text { selects beta- decays }
$$

Gamma Energy - A gamma ray from decay of a nuclear level is specified by its energy. This is a numeric selection parameter. Energies must be given in keV. For example

500-700 selects gamma rays from nuclear level decay between 500 and $700 \mathrm{keV}$.

Gamma Multipolarity - A decay gamma ray is characterized by its multipolarity. This is a character selection parameter. Electric radiation is given in the form E0, E1, E2, E3, etc.; magnetic radiation in the form M1, M2, M3, etc. For example

E1\& M2 selects gamma rays with an E1 component and no M2 component. 
Radiation - The radiation from a radioactive decay may be selected. This is a character selection parameter. The possible radiations and their character codes are

$$
\begin{aligned}
& \text { B- - beta- particles } \\
& \text { B+ - positrons } \\
& \text { E - electrons } \\
& \text { G - gamma rays } \\
& \text { A - alpha particles }
\end{aligned}
$$

Use these character codes to select the radiation type. For example

$$
\text { B+ } \quad \text { selects positrons }
$$

Radiation Energy - A decay radiation is characterized by its energy. In the case of beta radiation, this is the median energy of the beta spectrum. This is a numeric selection parameter. Energies must be given in $\mathrm{keV}$. For example

500-900 selects radiations with energies between 500 and 900 $\mathrm{keV}$.

Radiation Intensity - A decay radiation is characterized by its frequency per decay. This is a numeric selection parameter. Intensities must be given in percent (frequency per 100 decays). For example

80.- $\quad$ selects radiations with intensities greater than $80 \%$.

Thermal Data Type - Thermal neutron reaction data type may be selected. This is a character selection parameter. The possible data types and their character codes are

$$
\begin{aligned}
& \text { T }- \text { total cross section } \\
& \text { S }- \text { scattering cross section } \\
& \text { A }- \text { absorption cross section } \\
& \text { C - capture cross section } \\
& \text { F }- \text { fission neutron multiplicity }(\bar{\nu})
\end{aligned}
$$

Use these character codes to select the thermal neutron data type. For example

$$
\text { C@F } \quad \text { selects capture and fission cross sections. }
$$


Thermal Data Value - Thermal data may be selected by its value. This is a numeric selection parameter. Cross sections are given in barns and multiplicities as number per reaction. For example

$$
\text { 1.E+6- selects reactions with cross sections greater than or }
$$
equal to 1 million barns.

Resonance Integral - Resonance integrals may be selected by their value. This is a numeric selection parameter. Resonance integrals are given in barns. For example

-200.5 selects reactions with resonance integrals less than or equal to 200.5 barns.

\section{RETRIEVE}

When the data selection parameters have been supplied, the data base is searched and the selected data is stored. The number of records satisfying the parameter selection criteria is displayed. To output some or all of this data choose the SELECT option. The video mode display for the RETRIEVE option is given below. Note in the figure below that the RETRIEVE/RESTRICT option has been executed so that the current number of selected data records is less than the original (base) number of data records.

Data Output Menu Display in Video Environment

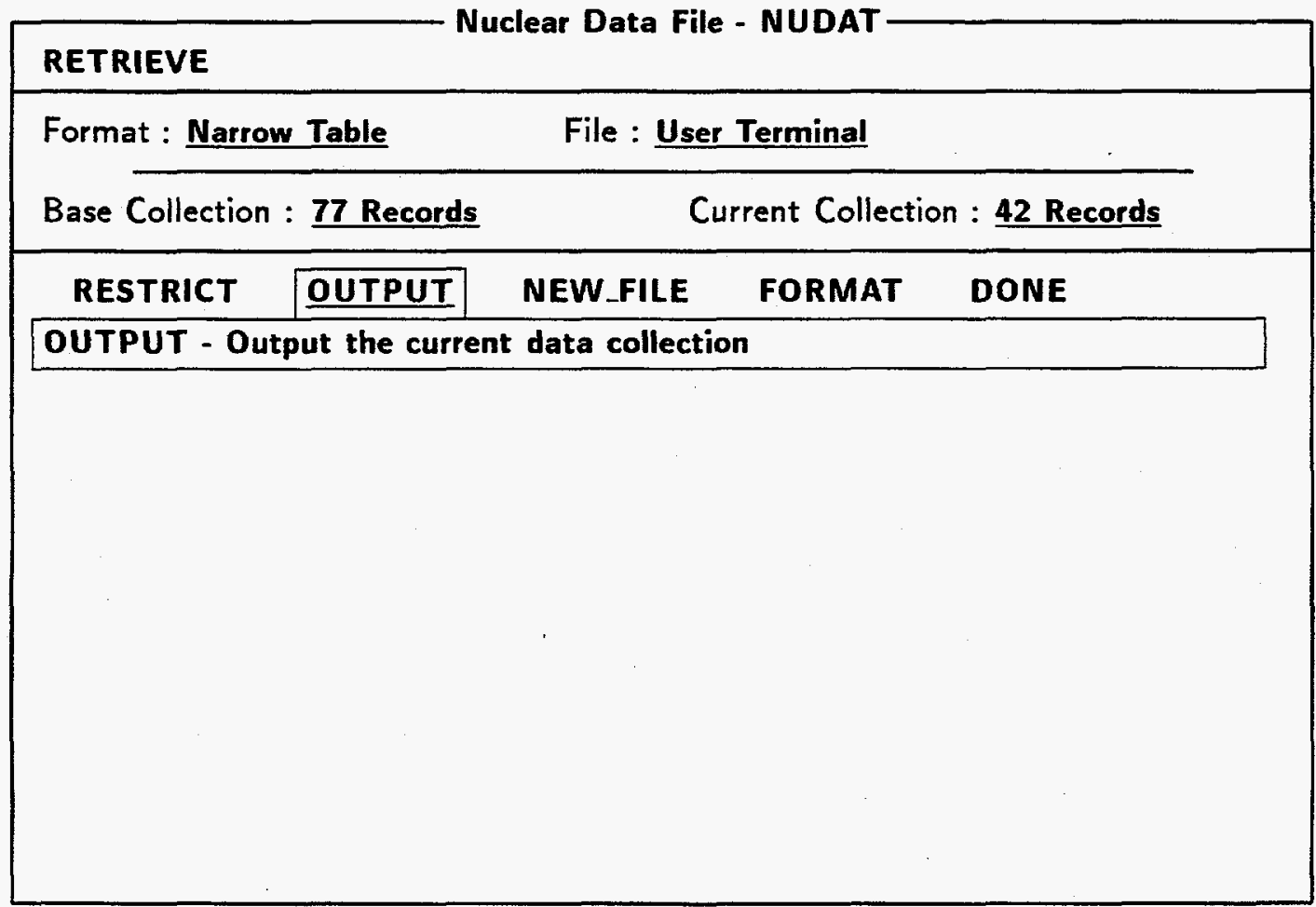




\subsection{RETRIEVE/RESTRICT}

A restricted subset of the data previously selected based on the initial values of the selection parameters may be chosen for display. The RETRIEVE/RESTRICT option accomplishes this task. The execution of this option is identical to the interaction used to specify the primary data selection criteria in both the video and the sequential mode. The input forms are initialized to the initial criteria. Choose and enter the revised parameter selection expressions desired. Make certain that the revised selection criteria for a parameter is not incompatible with the initial selection criteria. For example, if the initial selection for the mass number was between 132 and 135 and the restriction selection expression is less than 125 , then no data will meet the restricted criteria.

\subsection{RETRIEVE/OUTPUT}

This option will output the currently selected data record to the user's terminal or to a disk file in the selected format. The data may be sorted in a number of ways depending on the data type being output. The following table summarizes the sorting options available. The first option listed is the default.

\section{Levels}

Mass number, Proton number, Level energy

Level energy, Mass number, Proton number

\section{Gammas}

Mass number, Proton number, Level energy, Gamma energy Level energy, Mass number, Proton number, Gamma energy Gamma energy, Mass number, Proton number, Level energy

\section{Levels and Gammas}

Mass number, Proton number, Level energy, Gamma energy Level energy, Mass number, Proton number, Gamma energy Gamma energy, Mass number, Proton number, Level energy

WALLET CARDS

Mass number, Proton number, State energy, Half-life

\section{Decay Radiations}

Mass number, Proton number, Half-life, Radiation

Radiation energy, Mass number, Proton number

Radiation intensity, Radiation energy, Mass number, Proton number

\section{Neutron Data \\ Mass number, Proton number, State energy}

For the four data types given in the table above which have more than one sorting option, a menu for selecting the sorting order is presented in both the video and the sequential modes. For the other two data types where no sorting is supported, the output is 
immediately generated when the RETRIEVE/OUTPUT option is selected.

When output to disk, the retrieval proceeds uninterrupted in the format selected in the FORMAT option. A message indicating that the output to disk is in progress is displayed. This message disappears when the retrieval has been completed. When terminal output has been selected, in video mode, the output is scrolled whereas in sequential mode, it is paged.

Video

In video mode, the movement within the output of the data is controlled by four scrolling options in the menu:

FORWARD - to move forward a page,

BACKWARD - to move backward a page,

START - to move to the first page,

END - to move to the last page.

When one of the options is not logically possible because of the current position within the data output, then it does not appear in the menu. For example, FORWARD and END do not appear when the window is displaying the last page of the data. Selecting DONE indicates that the user has finished viewing the retrieval.

Sequential

In the sequential mode, the display is nearly identical to that of the video mode. The menu items are displayed at the bottom of the screen are are identical in both modes. Select a menu item by entering the first character of the item name.

Output Display for an Levels Retrieval in Video Environment

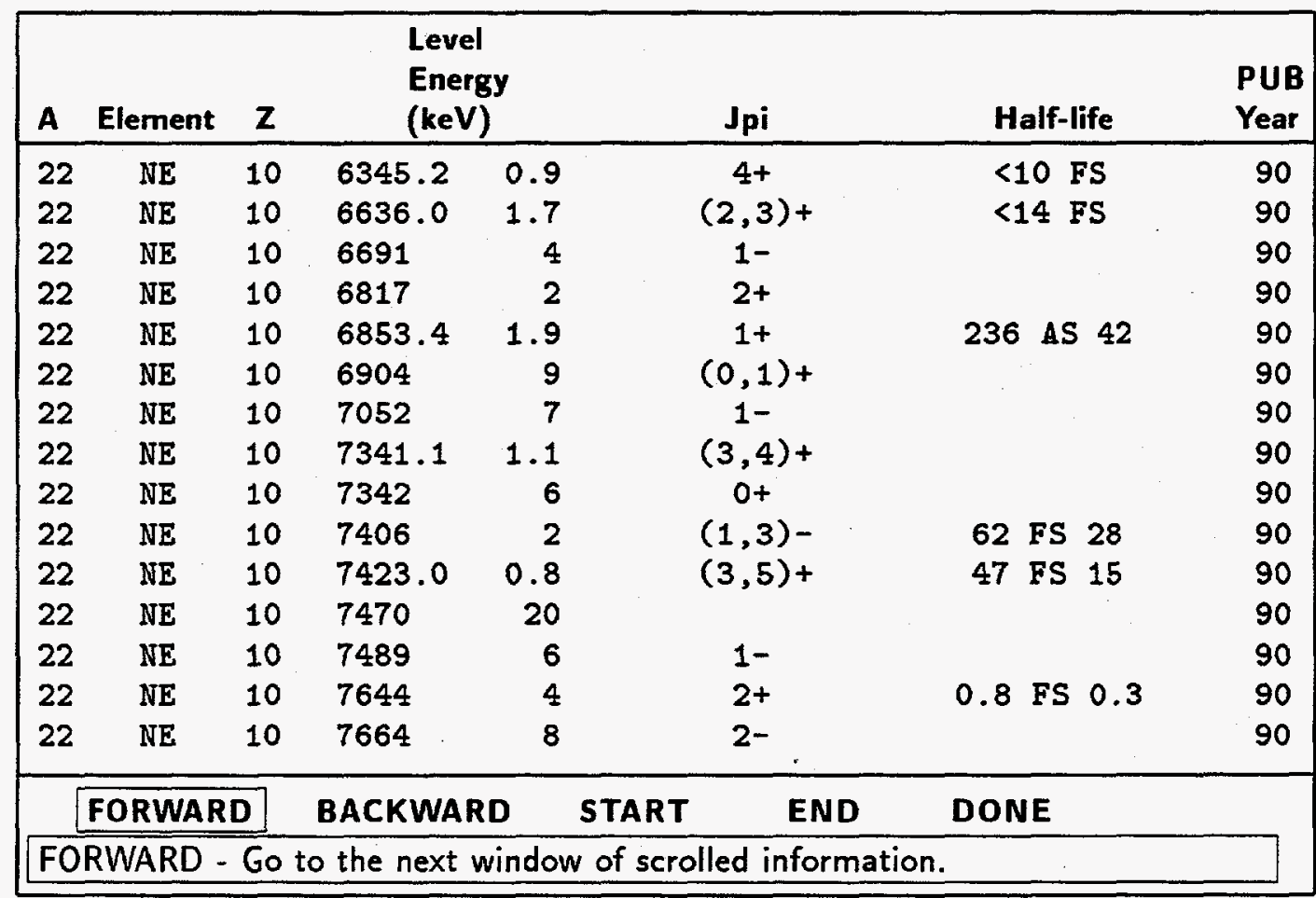




\subsection{RETRIEVE/SEND}

Use this option to download the data file containing the current retrieval. This option appears in the RETRIEVE menu only when a file of retrieved data exists. This option is is not available in the $\mathrm{PC}$ version.

\subsection{RETRIEVE/NEW_FILE}

Use this option to change where the retrieval output will go. This option is identical to the NEW FILE option described in section 8.

\subsection{RETRIEVE/FORMAT}

Use this option to change the format of the retrieval output. This option is identical to the FORMAT option described in section 7.

\section{FORMAT}

The output can be generated in one of two formats. This option permits the selection of the output format from the two choices. The default selection is Wide Table format for terminals with 132-character display capability or for disk output.

\section{Wide Table Format}

The Wide Table format is designed for 132 character line output. For terminal output, unless the user has specified that his output device is 132-character capable, the output will revert to the Narrow Table format.

\section{Narrow Table Format}

The Narrow Table format is a tabular output limited to 80 character output lines. In some cases, the number of fields output will be less than in the wide table format.

\section{NEW_FILE}

The output from the retrieval can be displayed on the user's terminal or stored in a disk file. This option can be used to change the currently selected output destination. The default destination is the user's terminal. The user's terminal can be indicated by specifying a file TT: or TTY : .

When this option is selected, a prompt appears and the user enters a new file specification. Entering a RETURN without a file specification is interpreted as selecting the user terminal for output. The video and sequential mode operation is the same. 


\section{MASS_STATUS}

The ENSDF data evaluations from which a large part of the data in this data base are derived, are carried out simultaneously for all nuclides with the same mass number (mass chain). When the evaluation for a mass chain has been completed, it is reviewed and then published in the Nuclear Data Sheets Journal or in the case of A less than 45, in Nuclear Physics. When submitted for publication in Nuclear Data Sheets, the evaluation is added to the ENSDF data base maintained by the National Nuclear Data Center of Brookhaven National Laboratory. In the case for $A$ less than 45 , there can be a significant delay between publication and entry into the data base since the evaluations are coded in ENSDF format by other evaluators after publication.

The MASS_STATUS option is designed to provide the user with information about the publication status of a mass chain. After selecting this option, the user is asked to supply a valid mass number. Information about the mass chain evaluation is then displayed on the terminal. The information includes the publication citation and the cutoff date for literature included in the publication. If a revised evaluation is available in prepublication form, it is so indicated. The user may select any number of mass chains in this option. If a blank mass number is entered the option terminates.

Video

The user enters a mass number and a display similar to the one below is output to the terminal. When finished with the display, depress the RETURN-key and then enter another mass number or the RETURN-key again to terminate the option.

Sequential

In this mode, after the user enters a mass number, the information appears on the terminal and looks just like the video mode display except that the mass number prompt appears immediately at the bottom of the screen. 
MASS_STATUS Display in Video Environment

Nuclear Data File - NUDAT

MASS_STATUS

Status for Mass 189

This evaluation was originally published in the Nuclear Data Sheets. Data may be incomplete or superceded by work published since the literature cutoff date. For references to later information on the mass chain, se日 the Nuclear Structure References data base.

Evaluation published in the Nuclear Data Sheets:

Volume:59 Page:869 Year:1990

ENSDF has been updated since its publication.

Literature cutoff date for this evaluation: 31-0ct-1991

Press any key to continue. 


\section{References}

1. Evaluated Nuclear Structure Data File (ENSDF) - a computer file of evaluated experimental nuclear structure data maintained by the National Nuclear Data Center, Brookhaven National Laboratory.

2. Nuclear Data Sheets - Academic Press, New York. Evaluations published by mass number for $A=45$ to 266 . See page ii of any issue for index to $A$-chains or http://www.dne.bnl.gov/ burrows/ensdf.

3. Nuclear Physics - North Holland Publishing Co., Amsterdam - Evaluations by F. Ajzenberg-Selove and by D. R. Tilley, H. R. Weller and C. M. Cheves for A=5 to 20 .

4. Energy Levels of $A=21-44$ Nuclei (VII), P. M. Endt, Nuclear Physics A521, 1 (1990).

5. Nuclear Wallet Cards, J. K. Tuli, 1995 and subsequent updates by J. K. Tuli from the Evaluated Nuclear Structure Data File (ENSDF)

6. The 1995 Update to the Atomic Mass Evaluation, G. Audi and A. H. Wapstra, Nucl. Phys. A595, 409 (1995).

7. The Program RADLIST, T. W. Burrows, Report BNL-NCS-52142 (1988)

8. Neutron Cross Sections, Vol. 1, Neutron Resonance Parameters and Thermal Cross Sections, Part A, Z=1-60, S.F. Mughabghab, M. Divadeenam, and N. E. Holden, Academic Press (1981)

9. Neutron Cross Sections, Vol. 1, Neutron Resonance Parameters and Thermal Cross Sections, Part B, Z=61-100, S.F. Mughabghab, Academic Press (1984)

10. Online Nuclear Data Service, C. L. Dunford and T. W. Burrows, Report NNDC/ONL95/10 (1995), Brookhaven National Laboratory 